\title{
Migration et système de santé
}

Jean Martin

jean.martin[at]saez.ch
A l'occasion du $125^{\mathrm{e}}$ anniversaire de la Policlinique médicale universitaire de Lausanne, ses responsables ont demandé à l'Institut d'histoire de la médecine et de la santé publique d'étudier les interactions entre migration et santé dans leur canton au cours des deux derniers siècles. Que ce soit du point de vue de mouvements d'étudiants et de professionnels, de facteurs de maladie, ou de personnes venues d'ailleurs après avoir passé par traumatismes et déracinements.

Migration et système de santé brosse un panorama des rapports entre la santé, la médecine, ceux qui la pratiquent, les pouvoirs publics et les mouvements de personnes; que ces personnes migrent parce
Mon intérêt personnel à sa lecture a été aiguisé par le fait que j'ai été, au service de l'Etat de Vaud, un «descendant» des protagonistes dont parle ce livre. Après des stages cliniques ici et huit ans passés outremer, je suis revenu au pays en 1976 pour intégrer le Service de la santé publique où je suis resté 27 ans (dont 17 comme médecin cantonal).

\section{Médecins, soignants et étudiants étrangers}

La première partie du livre m'a beaucoup parlé dans la mesure où j'ai vécu et parfois actionné les mécanismes à l'œuvre: questions et interventions auprès du Service sanitaire cantonal, du Conseiller d'Etat chargé de la santé qui prend l'avis du Conseil de

\section{Le livre brosse un panorama des rapports entre la santé, la médecine, ceux qui la pratiquent, les pouvoirs publics et les mouvements de personnes.}

qu'elles viennent ici en villégiature ou pour s'y faire soigner, ou qu'elles cherchent refuge ou travail. Qu'elles soient riches ou pauvres et souvent maltraitées par la vie.

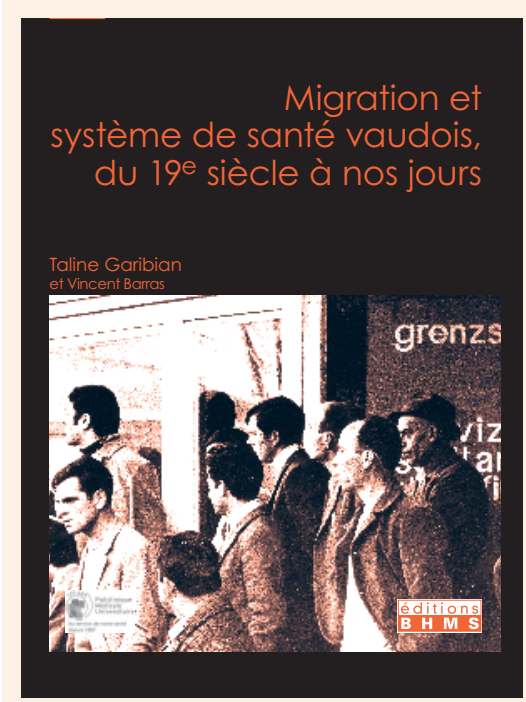

Taline Garibian, Vincent Barras

Migration et système de santé vaudois, du $19^{\text {e }}$ siècle à nos jours

Lausanne: Editions BHMS (Institut universitaire d'histoire de la médecine et de la santé publique); 2012 $\mathrm{XVI}+72$ pages, $30 \mathrm{CHF}$ ISBN 978-2-9700640-4-6 santé. Décisions y relatives. Relations avec les associations professionnelles, la société cantonale de médecine au premier chef. Craintes chroniques de pléthore médicale. J'ai été frappé aussi par des remarques et décisions du registre du racisme, notamment de l'antisémitisme; tout en sachant que, avant et jusqu'à la Seconde Guerre mondiale, l'antisémitisme était courant chez nous. Toutefois, on lit avec soulagement la manière dont en 1943 l'Université de Lausanne a refusé d'exclure des étudiants étrangers, notamment juifs italiens, disant qu'elle se place «au-dessus des partis, des nationalités et des contingences de race».

Pratiquement proche du racisme, une certaine xénophobie surgit dans les prises de position rapportées (dans les premières lignes du livre, on voit le Conseil fédéral, en 1921, recommander de ne pas accepter d'étrangers dont l'«affinité ethnique» est trop différente). Inquiétude qui tend à se justifier par des allégations de formation insuffisante, d'usage de méthodes différentes, de mauvaise intégration, en un mot d'«étrangeté». Crainte d'«invasion» (Überfremdung). Fonctionnement assez typique d'une corporation influente et proche des gens de pouvoir - ce qui en soi n'est pas inadmissible; une société est par définition un ensemble d'individus et groupes où s'exercent des influences.

Point d'actualité: au moment où ces lignes sont écrites, plusieurs associations professionnelles médicales suisses manifestent des inquiétudes compa- 
rables, s'agissant de confrères étrangers venant travailler ici, en particulier de l'Union européenne: on évoque une moins bonne formation de base et/ou post-graduée, la méconnaissance de la langue et de la culture régionale. On entend des propositions qui, mutatis mutandis, sont de la même veine qu'il y a 50, 100 ou 150 ans (proposition par exemple d'obligation de travailler d'abord plusieurs années de manière dépendante). Plus cela change... Au reste, faisant ces remarques, je ne prétends pas que les préoccupations en question soient sans fondement.

\section{Service de l'Etat, droit et justice...}

La lecture m'a amené à un questionnement qui m'habite de longue date, à propos d'attitudes de ceux qui sont venus avant nous et que nous tendons à juger de manière critique. Aurions-nous fait beaucoup mieux? Nous serions-nous élevés contre une position critiquable largement partagée par le public et les notables autour de nous? Cas échéant, aurionsnous eu le courage de faire œuvre de désobéissance civile?

Comme médecin cantonal, j'avais personnellement à formuler des préavis ou prendre des décisions dites de police sanitaire. Au nom des règles en vigueur, j'ai dû préaviser le retrait de l'autorisation de pratique de médecins d'origine étrangère qui avaient rendu service à notre canton durant des années mais dont on jugeait ne plus avoir besoin. Il m'est arrivé alors de dire que ma fonction m'amenait parfois à agir comme un salaud (sic). Découverte de ce que le droit n'est pas toujours la justice (parfois même, summum ius summa iniuria). Avec, scénario connu, le choix entre assumer malgré le malaise et s'efforcer de faire en sorte que ces situations se reproduisent le moins possible, ou rendre public son désaccord et démissionner ou être démissionné.

Pour être depuis longtemps en contact avec des collègues et organisations qui se préoccupent des personnes venues d'ailleurs, je sais avec quelle fréquence certains professionnels sont confrontés, avec leurs clients, à des situations où «ce n'est pas juste»: de ne pas croire celui-ci qui cherche refuge, de juger que ses raisons pour être accueilli sont insuffisantes, de dire que ses troubles de santé peuvent adéquatement être traités dans son pays d'origine ou sont du registre de la simulation, etc.

\section{Les maladies à travers les frontières}

Dans la période considérée, il y a toujours eu des étudiants étrangers à Lausanne. Au début du XX $\mathrm{X}^{\mathrm{e}}$ siècle, beaucoup venaient de Russie. Le livre rappelle des drames à large échelle, avec des dimensions racistes, liés aux épidémies de choléra dans ce pays, qui faisaient peur jusque chez nous - «St-Pétersbourg est à moins de soixante heures de Lausanne»...

S'agissant de maladies infectieuses, ma Guerre de Troie, avec beaucoup d'autres, a été l'épidémie VIH/ sida. C'est vers 1985 qu'on a pris conscience de ce qu'il s'agissait d'un grand problème de santé publique. Avec l'explosion de problématiques éthiques majeures, alimentées par le fait que la science n'avait pas de réponse efficace. D'où une floraison de propositions dont beaucoup menaient à des formes de stigmatisation, discrimination, limitation de la liberté des personnes concernées. Propositions auxquelles la Suisse a bien résisté grâce à des leaders de santé publique courageux, notamment à l'Office fédéral de la santé publique.

\section{Les migrants, actuellement}

On lit avec intérêt comment, au sortir de la Seconde Guerre, l'Académie Suisse des Sciences Médicales confie à la psychiatre zurichoise Maria Pfister-Ammende le soin de mener une enquête sur la santé mentale des internés; ses résultats viennent renforcer la perception des troubles dont ces derniers souffrent comme conséquences des atrocités de la guerre et des vicissitudes de leur parcours migratoire (notion qui n'a pas pris une ride!). Dans les années suivantes, une migration professionnelle peu qualifiée, venue d'abord d'Italie, va mettre au défi la communauté médicale. On peut évoquer ici ce que la profession appelait il y a un demi-siècle le «syndrome transalpin», dénomination péjorative des comportements jugés pusillanimes des travailleurs migrants italiens (des allégations de ce type se voient aujourd'hui à propos de demandes à l'assurance-invalidité par des personnes issues de l'immigration).

L'influence du milieu, donnée majeure de santé publique, retient alors l'attention. L'intérêt porté aux caractéristiques particulières des troubles de santé des migrants est d'abord le fait des psychiatres. Le Dr Jean-Louis Villa, de Lausanne, en vient à estimer que «son activité relève moins du traitement de pathologies psychiques que d'un travail de régulation sociale», «que c'est à chaque citoyen suisse de participer au bon accueil des travailleurs». Toutes les notions pertinentes ne sont pas d'aujourd'hui.

Ceci, il convient de le noter, à une époque où on pensait qu'il y a une (seule) bonne manière de se comporter si on se sent malade, vis-à-vis de soi et visà-vis du docteur ou de l'hôpital... alors qu'il n'y a jamais qu'une bonne manière. Le besoin est maintenant largement reconnu de compétences cliniques transculturelles adéquates.

En guise de conclusion: dans la relation avec et le traitement de patients migrants, dans la lutte contre les maladies infectieuses, dans les tâches de police sanitaire, grands sujets de cet ouvrage, les clés de l'action «juste», du mieux qu'on peut faire (parfois du moins mal), sont à mon sens un mélange de connaissances scientifiques et de compétences cliniques, d'expérience, d'attention au cadre juridicosocial mais aussi de respect des personnes, de compassion, de bon sens enfin. 